\title{
Comment on "Existence of Einstein static universes and their stability in fourth-order theories of gravity"
}

\author{
John Miritzis \\ Department of Marine Sciences, University of the Aegean \\ University Hill, Mytilene 81100, Greece \\ E-mail: imyr@aegean.gr \\ (Dated: October 30, 2018)
}

\begin{abstract}
It is argued that the solution space of the equation determining the class of $f(R)$ theories which admit an Einstein static universe should be broadened by including the algebraic roots.
\end{abstract}

PACS numbers: $04.50 . \mathrm{Kd}, 98.80 . \mathrm{Jk}$

In a recent paper, Goswami, Goheer and Dunsby (GGD) study the existence of Einstein static universes in the context of higher order theories of gravity [1]. They consider theories derived from a Lagrangian density

$$
L=f(R)-2 \Lambda+L_{m}
$$

in the Einstein static universe. The field equations imply Eq. (14) in Ref. [1], namely,

$$
R f^{\prime}-\frac{3}{2}(1+w) f+3 \Lambda(1+w)=0,
$$

where $w \in[-1,1]$ is the parameter in the equation of state, $p=w \rho$. Equation (1) has been discovered several times (cf [2] for a brief discussion of static solutions). GGD argue that the only functions $f(R)$ admitting the existence of an Einstein static universe are solutions of the differential equation (1) and therefore, have the form

$$
f(R)=2 \Lambda+K R^{\frac{3}{2}(1+w)}+L_{m} .
$$

According to this analysis, the solution (2) leaves no room for the theory derived from the Lagrangian density

$$
L=R+\beta R^{2}+L_{m},
$$

to admit the Einstein static universe as a solution. However, in a previous investigation it was found that this is not the case [3]. More precisely, without making use of expansion-normalized variables it is shown in Ref. [3] that the dynamical system has an unstable equilibrium of saddle type corresponding to the Einstein static universe. What is most interesting is that the effective cosmological constant is not assumed from the beginning, but is provided by the curvature equilibrium

$$
R_{*}=\frac{1+3 w}{\beta(1-3 w)}
$$

in the range $-\frac{1}{3}<w<\frac{1}{3}$.

In the conclusions of Ref. [1], GGD consider as surprising the result that only Lagrangians of the form $f(R)=a+b R^{c}$ admit an Einstein static space and furthermore comment: "This result differs from some recent work [36] (Ref. [4] in this comment), where the stability of the Einstein static solution was investigated for a number of types of $f(R)$ theories which appear not to fall into the class discovered here".

The existence of the Einstein static universe in $f(R)$ theories which do not to fall into the class (2), relies on the interpretation of (11). In fact, the solution (2) to the equation (11) is not the only possibility. Given an arbitrary differentiable function $f$, Eq. (11) can be regarded as an algebraic equation to be solved for $R$. Denoting the resulting roots by $\rho_{1}, \rho_{2}, \ldots$ one obtains a whole series of Einstein spaces, each having a constant scalar curvature $\rho_{i}$ (see for example the comments in Ref. [5] and for a thorough analysis see Ref. [6]). In view of the above interpretation, it is easy to check that substituting $f(R)=R+\beta R^{2}$ in (10) with $\Lambda=0$, the scalar curvature takes the constant value given by (3), exactly as was shown in Ref. [3].
[1] R. Goswami, N. Goheer and P.K.S. Dunsby, Phys. Rev. D 78, 044011 (2008).

[2] J.D. Barrow and A. Ottewill, J. Phys. A 1635 (1983).

[3] J. Miritzis, Gen. Rel. Grav. 41, 49 (2009). arXiv:0708.1396

[4] C.G. Böhmer, L. Hollenstein, and F.S.N. Lobo, Phys. Rev. D 76, 084005 (2007).
[5] S. Cotsakis, J. Miritzis and L. Querella, J. Math. Phys. 40, 3063 (1999).

[6] M. Ferraris, M. Francaviglia and I. Volovich, Class. Quant. Grav. 11, 1505 (1994). 